\title{
Inverse spectral problems for first order integro-differential operators
}

\section{Vjacheslav Yurko*}

\section{"Correspondence:} yurkova@info.sgu.ru Department of Mathematics, Saratov State University, Astrakhanskaya 83, Saratov, 410012 Russia

\section{Springer}

\begin{abstract}
Inverse spectral problems are studied for the first order integro-differential operators on a finite interval. Properties of spectral characteristic are established, and the uniqueness theorem is proved for this class of inverse problems.
\end{abstract}

MSC: 47G20; 45J05; 44A15

Keywords: integro-differential operators; inverse spectral problems; uniqueness theorem

\section{Introduction}

The paper is devoted to studying inverse spectral problems for integro-differential operators of the form

$$
\ell y:=i y^{\prime}(x)+R(x) \int_{0}^{x} V(t) y(t) d t .
$$

Inverse problems of spectral analysis consist in recovering operators from their spectral characteristics. Such problems often appear in mathematics, mechanics, physics, electronics, geophysics, meteorology and other branches of natural sciences. Inverse problems also play an important role in solving nonlinear evolution equations in mathematical physics. Interest in this subject has been increasing permanently because of the appearance of new important applications, and nowadays the inverse problem theory is being developed intensively all over the world. The greatest success in the inverse problem theory has been achieved for the Sturm-Liouville operator (see, e.g., [1-3]) and afterwards for higher order differential operators [4-6] and other classes of differential operators. For integro-differential and other classes of nonlocal operators, inverse problems are more difficult to investigate, and the main classical methods (transformation operator method and the method of spectral mappings) either are not applicable to them or require essential modifications; and for such operators, the general inverse problem theory does not exist. At the same time, nonlocal and, in particular, integro-differential operators are of great interest, because they have many applications (see, e.g., [7]). We note that some aspects of inverse problems for integro-differential and integral operators were studied in [8-12] and other works. In the present paper, we study the inverse spectral problem for the first order integro-differential operator $\ell$ on a finite interval. Properties of spectral character-

(c) The Author(s) 2017. This article is distributed under the terms of the Creative Commons Attribution 4.0 International License (http://creativecommons.org/licenses/by/4.0/), which permits unrestricted use, distribution, and reproduction in any medium, provided you give appropriate credit to the original author(s) and the source, provide a link to the Creative Commons license, and indicate if changes were made. 
istics are established, and the uniqueness theorem is proved for the inverse problem of recovering the function $R(x)$ and $V(x)$ from the given spectral data.

\section{Preliminary information}

Consider the integro-differential equation

$$
\ell y:=i y^{\prime}(x)+R(x) \int_{0}^{x} V(t) y(t) d t=\lambda y(x), \quad x \in[0, \pi],
$$

where $R(x), V(t)$ are continuous complex-valued functions, and

$$
R(\pi-x) \sim C_{\alpha} x^{\alpha}, \quad V(x) \sim D_{\beta} x^{\beta}, \quad x \rightarrow+0, \quad C_{\alpha} D_{\beta} \neq 0 .
$$

Let $\varphi(x, \lambda)$ be the solution of Eq. (1) with the condition $\varphi(0, \lambda)=1$. Then the following representation holds (see [3]):

$$
\varphi(x, \lambda)=\exp (-i \lambda x)+\int_{0}^{x} K(x, t) \exp (-i \lambda t) d t
$$

where $K(x, t)$ is a continuous function, and $K(x, 0)=0$. Denote

$$
\Pi_{+}:=\{\lambda: \operatorname{Im} \lambda \geq 0\}, \quad \Pi_{-}^{\delta}:=\{\lambda: \arg \lambda \in[\pi+\delta, 2 \pi-\delta]\} .
$$

It follows from (2) that for $|\lambda| \rightarrow \infty$ uniformly in $x \in[0, \pi]$ :

$$
\left.\begin{array}{l}
\varphi^{(v)}(x, \lambda)=(-i \lambda)^{v} \exp (-i \lambda x)(1+o(1)), \quad \lambda \in \Pi_{+}, v=0,1, \\
\varphi^{(v)}(x, \lambda)=o\left(\lambda^{\nu}\right), \quad \lambda \in \Pi_{-}^{\delta}, v=0,1 .
\end{array}\right\}
$$

Denote

$$
\varphi_{\nu}(x, \lambda):=\frac{1}{\nu !} \frac{\partial^{v} \varphi(x, \lambda)}{\partial \lambda^{v}}, \quad v \geq 0, \quad \Delta(\lambda):=\varphi(\pi, \lambda) .
$$

The function $\Delta(\lambda)$ is entire in $\lambda$ of exponential type, and its zeros $\Lambda:=\left\{\lambda_{n}\right\}_{n \geq 1}$ (counting with multiplicities) coincide with the eigenvalues of the boundary value problem $L=L(R, V)$ for Eq. (1) with the condition $y(\pi)=0$. Let $m_{n}$ be the multiplicity of $\lambda_{n}$ $\left(\lambda_{n}=\lambda_{n+1}=\cdots=\lambda_{n+m_{n}-1}\right)$. Denote

$$
S:=\left\{n: n-1 \in \mathbf{N}, \lambda_{n-1} \neq \lambda_{n}\right\} \cup\{1\}, \quad s_{n+v}(x):=\varphi_{\nu}\left(x, \lambda_{n}\right), \quad n \in S, v=\overline{m_{n}-1} .
$$

The functions $\left\{s_{n}(x)\right\}_{n \geq 1}$ are eigen and associated functions for $L$.

Example 1 Let $\lambda_{1}=\lambda_{2}<\lambda_{3}<\lambda_{4}=\lambda_{5}=\lambda_{6}<\lambda_{7}<\lambda_{8}<\cdots$. Then $S=\{1,3,4,7,8, \ldots\}$, $s_{1}(x)=\varphi_{0}\left(x, \lambda_{1}\right), s_{2}(x)=\varphi_{1}\left(x, \lambda_{1}\right), s_{3}(x)=\varphi_{0}\left(x, \lambda_{3}\right), s_{4}(x)=\varphi_{0}\left(x, \lambda_{4}\right), s_{5}(x)=\varphi_{1}\left(x, \lambda_{4}\right), s_{6}(x)=$ $\varphi_{2}\left(x, \lambda_{4}\right), s_{7}(x)=\varphi_{0}\left(x, \lambda_{7}\right), \ldots$

Let the function $\eta(x, \lambda)$ be the solution of the problem

$$
i \eta^{\prime}(x, \lambda)-R(x) \int_{x}^{\pi} V(t) \eta(t, \lambda) d t+R(x)=\lambda \eta(x, \lambda), \quad \eta(\pi, \lambda)=0 .
$$


Denote $\theta(x, \lambda):=\eta(\pi-x, \lambda)$. Then

$$
i \theta^{\prime}(x, \lambda)+R_{0}(x) \int_{0}^{x} V_{0}(t) \theta(t, \lambda) d t-R_{0}(x)=-\lambda \theta(x, \lambda), \quad \theta(0, \lambda)=0,
$$

where $R_{0}(x):=R(\pi-x), V_{0}(x):=V(\pi-x)$. It follows from (5) that

$$
\theta(x, \lambda)=\int_{0}^{x} g(x, t, \lambda) R_{0}(t) d t
$$

where $g(x, t, \lambda)$ is Green's function of the Cauchy problem, and

$$
i g_{x}(x+t, t, \lambda)-\lambda g(x+t, t, \lambda)+R(x+t) \int_{0}^{x} V(\tau+t) g(\tau+t, t, \lambda) d \tau=0, \quad g(t, t, \lambda)=-i
$$

and consequently, $g(x+t, t, \lambda)=-i \varphi(x, \lambda ; t)$, where $\varphi(x, \lambda ; t)$ is the solution of the Cauchy problem

$$
i \varphi^{\prime}(x, \lambda ; t)+R(x+t) \int_{0}^{x} V(\tau+t) \varphi(\tau, \lambda ; t) d \tau=\lambda \varphi(x, \lambda ; t), \quad \varphi(0, \lambda ; t)=1 .
$$

In view of (2) we get

$$
\varphi(x, \lambda ; t)=\exp (-i \lambda x)+\int_{0}^{x} K(x, \tau ; t) \exp (-i \lambda \tau) d \tau
$$

where $K(x, \tau ; t)$ is a continuous function. This yields

$$
g(x, t, \lambda)=-i \exp (-i \lambda(x-t))-i \int_{0}^{x-t} K(x-t, \tau ; t) \exp (-i \lambda \tau) d \tau
$$

Substituting (7) into (6), we obtain

$$
\theta(x, \lambda)=\int_{0}^{x} P(x, t) \exp (i \lambda t) d t
$$

where

$$
P(x, t)=-i R_{0}(x-t)-i \int_{0}^{x-t} R_{0}(\tau) K(x-\tau, t ; \tau) d \tau .
$$

Clearly, $P(x, x)=-i R_{0}(0), P(x, 0)=-i R_{0}(x)$. Using (8)-(9) and (4), we conclude that for $|\lambda| \rightarrow \infty$ uniformly in $x \in[0, \pi]$ :

$$
\left.\begin{array}{l}
\eta^{(v)}(x, \lambda)=o\left(\lambda^{v}\right), \quad \lambda \in \Pi_{+}, v=0,1, \\
\eta^{(v)}(x, \lambda)=o\left(\lambda^{v} \exp (i \lambda(\pi-x))\right), \quad \lambda \in \Pi_{-}^{\delta}, v=0,1 .
\end{array}\right\}
$$

Denote

$$
\Delta_{0}(\lambda):=1-\int_{0}^{\pi} V(t) \eta(t, \lambda) d t
$$


Using (4) and (11), we calculate

$$
i \eta^{\prime}(x, \lambda)+R(x)\left(\Delta_{0}(\lambda)+\int_{0}^{x} V(t) \eta(t, \lambda) d t\right)=\lambda \eta(x, \lambda), \quad \eta(\pi, \lambda)=0 .
$$

In particular, it follows from (12) that the zeros of the entire function $\Delta_{0}(\lambda)$ coincide with the zeros of $\Delta(\lambda)$, and multiplicities of zeros of $\Delta_{0}(\lambda)$ are not more than multiplicities of zeros of $\Delta(\lambda)$. Therefore the function $\Delta(\lambda) / \Delta_{0}(\lambda)$ is entire in $\lambda$ of exponential type. Denote $\Delta_{1}(\lambda):=\Delta_{0}(\lambda) \exp (-i \lambda \pi)$. Using (8), (9) and (11), by standard arguments (see, e.g., [3]), we obtain that for $|\lambda| \rightarrow \infty$, the following asymptotical formulae hold:

$$
\left.\begin{array}{l}
\Delta_{1}(\lambda)=\exp (-i \lambda \pi)(1+o(1)), \quad \lambda \in \Pi_{+}, \\
\Delta_{1}(\lambda)=B \lambda^{-\gamma-1}(1+o(1)), \quad \lambda \in \Pi_{-}^{\delta},
\end{array}\right\}
$$

where $B \neq 0, \gamma:=\alpha+\beta+1$. The function $F(\lambda):=\Delta(\lambda) / \Delta_{1}(\lambda)$ is entire in $\lambda$ of exponential type. By virtue of (3),

$$
\left.\begin{array}{l}
\Delta(\lambda)=\exp (-i \lambda \pi)(1+o(1)), \quad \lambda \in \Pi_{+}, \\
\Delta(\lambda)=o(1), \quad \lambda \in \Pi_{-}^{\delta} .
\end{array}\right\}
$$

Together with (13) this yields that $F(\lambda) \equiv 1$, i.e., $\Delta(\lambda) \equiv \Delta_{1}(\lambda)$ or

$$
\Delta(\lambda) \equiv \Delta_{0}(\lambda) \exp (-i \lambda \pi)
$$

Denote

$$
\eta_{v}(x, \lambda):=\frac{1}{\nu !} \frac{\partial \eta(x, \lambda)}{\partial \lambda^{v}}, \quad v \geq 0, \quad \psi_{n+v}(x):=\eta_{v}\left(x, \lambda_{n}\right), \quad n \in S, v=\overline{m_{n}-1} .
$$

The functions $\left\{\psi_{n}(x)\right\}_{n \geq 1}$ are eigen and associated functions for the boundary value problem $L$, and

$$
\psi_{n+v}(x)=\sum_{j=0}^{v} \beta_{n+v-j} s_{n+j}(x), \quad n \in S, v=\overline{m_{n}-1}
$$

The coefficients $\left\{\beta_{n}\right\}_{n \geq 1}$ are called Levinson's weight numbers, and the data $\left\{\lambda_{n}, \beta_{n}\right\}_{n \geq 1}$ are called the spectral data for the boundary value problem $L$. We will consider the following inverse problem.

Inverse problem 1 Given the spectral data $\left\{\lambda_{n}, \beta_{n}\right\}_{n \geq 1}$, construct $R$ and $V$.

\section{The uniqueness theorem}

Below we will assume that $R(x) \neq 0$ a.e. on $(0, \pi)$. If this condition does not hold, then the specification of the spectral data does not uniquely determine $L$ (see Example 2).

Let us formulate the uniqueness theorem for this inverse problem. For this purpose, together with $L$ we consider the boundary value problem $\tilde{L}:=L(\tilde{R}, \tilde{V})$ of the same form but with different functions $\tilde{R}(x), \tilde{V}(t)$. We agree that in what follows if a certain symbol $\alpha$ denotes an object related to $L$, then $\tilde{\alpha}$ will denote the analogous object related to $\tilde{L}$. 
Theorem 1 Let $\left\{\tilde{\lambda}_{n}, \tilde{\beta}_{n}\right\}$ be the spectral data for the problem $\tilde{L}=L(\tilde{R}, \tilde{V})$. If $\lambda_{n}=\tilde{\lambda}_{n}, \beta_{n}=\tilde{\beta}_{n}$ for all $n \geq 1$, then $R(x) \equiv \tilde{R}(x), V(x) \equiv \tilde{V}(x), x \in[0, \pi]$.

Proof Using (14)-(15) and Hadamard's factorization theorem, we get $\Delta_{0}(\lambda) \equiv \tilde{\Delta}_{0}(\lambda)$. Taking (16) into account, we deduce that the functions

$$
A_{j}(x, \lambda)=\left(\Delta_{0}(\lambda)\right)^{-1} \exp (i \lambda x)\left(\tilde{\varphi}(x, \lambda) \eta^{(j-1)}(x, \lambda)-\tilde{\eta}(x, \lambda) \varphi^{(j-1)}(x, \lambda)\right), \quad j=1,2,
$$

are entire in $\lambda$ of exponential type. Taking (3), (10) and (13) into account, we obtain for $|\lambda| \rightarrow \infty$

$$
\begin{aligned}
& A_{1}(x, \lambda)=o(1), \quad A_{2}(x, \lambda)=o(\lambda), \quad \lambda \in \Pi_{+}, \\
& A_{1}(x, \lambda)=o\left(\lambda^{\gamma+1}\right), \quad A_{2}(x, \lambda)=o\left(\lambda^{\gamma+2}\right), \quad \lambda \in \Pi_{-}^{\delta},
\end{aligned}
$$

and consequently,

$$
A_{1}(x, \lambda) \equiv 0, \quad A_{2}(x, \lambda) \equiv A(x)
$$

where the function $A(x)$ does not depend on $\lambda$. In particular, (17) yields

$$
\begin{aligned}
& \tilde{\varphi}(x, \lambda) \eta(x, \lambda) \equiv \tilde{\eta}(x, \lambda) \varphi(x, \lambda), \\
& \tilde{\varphi}(x, \lambda) \eta^{\prime}(x, \lambda)-\tilde{\eta}(x, \lambda) \varphi^{\prime}(x, \lambda) \equiv A(x) \Delta_{0}(\lambda) \exp (-i \lambda x) .
\end{aligned}
$$

Similarly, we obtain

$$
\varphi(x, \lambda) \eta^{\prime}(x, \lambda)-\eta(x, \lambda) \varphi^{\prime}(x, \lambda) \equiv A^{*}(x) \Delta_{0}(\lambda) \exp (-i \lambda x)
$$

where $A^{*}(x)$ does not depend on $\lambda$. Using (18) we calculate

$$
\tilde{\varphi}(x, \lambda)\left(\varphi(x, \lambda) \eta^{\prime}(x, \lambda)-\eta(x, \lambda) \varphi^{\prime}(x, \lambda)\right)=\varphi(x, \lambda)\left(\tilde{\varphi}(x, \lambda) \eta^{\prime}(x, \lambda)-\tilde{\eta}(x, \lambda) \varphi^{\prime}(x, \lambda)\right) .
$$

Together with (19)-(20) this yields

$$
\tilde{\varphi}(x, \lambda) A^{*}(x) \equiv \varphi(x, \lambda) A(x) .
$$

Taking (3) into account, we conclude that $A(x) \equiv A^{*}(x)$, and

$$
(\tilde{\varphi}(x, \lambda)-\varphi(x, \lambda)) A(x) \equiv 0 .
$$

Furthermore, using (18), (19) and Eqs. (1) and (4), we infer

$$
\begin{aligned}
i A & (x) \Delta_{0}(\lambda) \exp (-i \lambda x) \\
& =i \tilde{\varphi}(x, \lambda) \eta^{\prime}(x, \lambda)-i \tilde{\eta}(x, \lambda) \varphi^{\prime}(x, \lambda) \\
& =\tilde{\varphi}(x, \lambda)\left(R(x) \int_{x}^{\pi} V(t) \eta(t, \lambda) d t-R(x)\right)+\tilde{\eta}(x, \lambda) R(x) \int_{0}^{x} V(t) \varphi(t, \lambda) d t .
\end{aligned}
$$


Hence, for $|\lambda| \rightarrow \infty, \lambda \in \Pi_{+}$, we get $A(x) \equiv i R(x)$. In view of (21), one has

$$
(\tilde{\varphi}(x, \lambda)-\varphi(x, \lambda)) R(x) \equiv 0
$$

Since $R(x) \neq 0$ a.e. on $(0, \pi)$, it follows from (22) that

$$
\tilde{\varphi}(x, \lambda) \equiv \varphi(x, \lambda)
$$

By virtue of (18),

$$
\tilde{\eta}(x, \lambda) \equiv \eta(x, \lambda)
$$

Then, according to (4),

$$
R(x)-\tilde{R}(x)=R(x) \int_{x}^{\pi} V(t) \eta(t, \lambda) d t-\tilde{R}(x) \int_{x}^{\pi} \tilde{V}(t) \eta(t, \lambda) d t
$$

For $|\lambda| \rightarrow \infty$ this yields $R(x) \equiv \tilde{R}(x), x \in[0, \pi]$, and consequently $V(x) \equiv \tilde{V}(x), x \in[0, \pi]$. Theorem 1 is proved.

Example 2 Fix $a \in(0, \pi)$. Let $R(x) \equiv 0$ for $x \in[0, a]$ and $R(x) \neq 0$ for $x \in(a, \pi)$. Put $\tilde{R}(x) \equiv$ $R(x)$ for $x \in[0, \pi]$, and choose $V(t), \tilde{V}(t)$ such that $V(t) \equiv \tilde{V}(t)$ for $t \in(a, \pi)$, and $V(t) \neq$ $\tilde{V}(t)$ for $t \in[0, a]$. Then $\tilde{\varphi}(x, \lambda) \equiv \varphi(x, \lambda)$ and $\tilde{\eta}(x, \lambda) \equiv \eta(x, \lambda)$; hence $\tilde{\lambda}_{n}=\lambda_{n}, \tilde{\beta}_{n}=\beta_{n}$ for all $n \geq 1$.

\section{Acknowledgements}

This work was supported by Grant 17-11-01193 of the Russian Science Foundation.

Funding

This work was supported by Grant 17-11-01193 of the Russian Science Foundation.

\section{Abbreviations}

Not applicable.

Availability of data and materials

Not applicable.

Ethics approval and consent to participate

Not applicable.

Competing interests

No potential conflict of interest was reported by the author.

Consent for publication

Not applicable.

Author's contributions

All authors read and approved the final manuscript.

Author's information

Vjacheslav Yurko is Full Professor, Head of the Faculty of Mathematical Physics.

\section{Publisher's Note}

Springer Nature remains neutral with regard to jurisdictional claims in published maps and institutional affiliations. 
References

1. Marchenko, VA: Sturm-Liouville Operators and Their Applications. Naukova Dumka, Kiev (1977) English transl. Birkhäuser, Basel, 1986

2. Levitan, BM: Inverse Sturm-Liouville Problems. Nauka, Moscow (1984) English transl., VNU Sci. Press, Utrecht, 1987

3. Freiling, G, Yurko, VA: Inverse Sturm-Liouville Problems and Their Applications. Nova Science Publishers, New York (2001)

4. Beals, R, Deift, P, Tomei, C: Direct and Inverse Scattering on the Line. Math. Surveys and Monographs, vol. 28. Am. Math. Soc., Providence (1988)

5. Yurko, VA: Method of Spectral Mappings in the Inverse Problem Theory. Inverse and III-Posed Problems Series. VSP, Utrecht (2002)

6. Yurko, VA: Inverse Spectral Problems for Differential Operators and Their Applications. Gordon \& Breach, New York (2000)

7. Lakshmikantham, V, Rao, HRM: Theory of Integro-Differential Equations. Stability and Control: Theory and Applications, vol. 1. Gordon \& Breach, New York (1995)

8. Yurko, VA: An inverse problem for integro-differential operators. Mat. Zametki 50(5), 134-146 (1991) (Russian); English transl. in Math. Notes 50(5-6), 1188-1197 (1991)

9. Kuryshova, Yu: An inverse spectral problem for differential operators with integral delay. Tamkang J. Math. 42(3), 295-303 (2011)

10. Buterin, SA: On the reconstruction of a convolution perturbation of the Sturm-Liouville operator from the spectrum Differ. Uravn. (Minsk) 46, 146-149 (2010) (Russian); English transl. in Diff. Equ. 46, 150-154 (2010)

11. Yurko, VA: An inverse problem for integral operators. Mat. Zametki 37(5), 690-701 (1985) (Russian); English transl. in Math. Notes 37(5-6), 378-385 (1985)

12. Buterin, SA, Choque Rivero, AE: On inverse problem for a convolution integro-differential operator with Robin boundary conditions. Appl. Math. Lett. 48, 150-155 (2015)

\section{Submit your manuscript to a SpringerOpen ${ }^{\circ}$ journal and benefit from:}

- Convenient online submission

- Rigorous peer review

- Open access: articles freely available online

- High visibility within the field

- Retaining the copyright to your article 